\title{
DNA Primase Large Subunit
}

National Cancer Institute

\section{Source}

National Cancer Institute. DNA Primase Large Subunit. NCI Thesaurus. Code C38619.

DNA primase large subunit (509 aa, $\sim 59 \mathrm{kDa}$ ) is encoded by the human PRIM2 gene. This protein plays a role in the synthesis of small RNA primers to resolve the Okazaki fragments made during discontinuous DNA replication. 